IP Periodica Polytechnica

Transportation Engineering

43(4), pp. 211-217, 2015

DOI: 10.3311/PPtr.7845

Creative Commons Attribution (i)

RESEARCH ARTICLE

\section{Developing a New Logistics Based Model and Pilot System for Construction}

\author{
András Gyimesi ${ }^{1 *}$, Gábor Bohács ${ }^{1}$
}

Received 03 November 2014; accepted after revision 26 January 2015

\begin{abstract}
This paper presents the development of a new construction logistics model with a new point of view: we want to integrate related experiences from the practice of other industries with the specialties of construction logistics processes. It gives a detailed description on the proposed model. Furthermore, the basics of a demonstration physical test system will be described as well. The development introduced in this paper is a part of a Hungarian Government-financed research project (reference no. KTIA_AIK_12-1-2013-0009). The whole budget (c.a. 1.36 Million EUR) of the research is provided by the KTIA fund.
\end{abstract}

\section{Keywords}

Construction logistics, modeling, construction process management, process control, adaptive simulation, pilot system

\footnotetext{
${ }^{1}$ Department of Material Handling and Logistics System Faculty of Transport Engineering,

Budapest University of Technology and Economics

H-1521 Budapest, P.O.B. 91, Hungary
}

*Corresponding author, e-mail: andras.gyimesi@logisztika.bme.hu

\section{Introduction}

As the construction industry has one of the biggest influences on the economy, it has to be provided with similar initial conditions as the other economically important sectors. In order to create an effective process and control system, we need a good model of the technological and logistic processes. We have to understand the effects of one change or another that we have to make progress. There are several industries, where modeling is used as an everyday practice of developers. For example, there are several boxed software solutions for warehouse designers to simulate the material handling processes / material flow in their facilities. We may have to try several variants of different modeling methods for the construction industry and its logistics, but we have to recognize that construction processes are so unique: it has so many independent environmental variables and each element lasts only for a short period of time. It means that no real recurrence can be found. The processes are similar to the military ones: there are so many impacting factors from outside of the system, and we have a short interval to be successful.

\section{Researches examined}

This area of research gained momentum in the last 10 years with the progress of information technology, so we have several tentative modeling methods with several different points of view. For example, an interesting geometry-based model was invented for construction processes at CIFE (Stanford University). (Akbas, 2014)

The base of the modeling procedure is to slice the work field into elemental pieces (e.g. 3D triangles), and then we can examine the process as a summation of elemental process elements from those elemental layouts. For those elemental processes, we can use a reduced model that we can simulate as networks, shown in Fig. 1. But the network of the elemental queuing networks will not be as flexible and adaptive to modeling the changes of the main process: the complex network has too many variables to let us follow all the effects instantly, if we have a conscious change or unexpected macro-effects.

In the paper of Jan Tulke and Jochen Han from the Bauhaus University (Tulke and Han, 2007) we can find instances about 


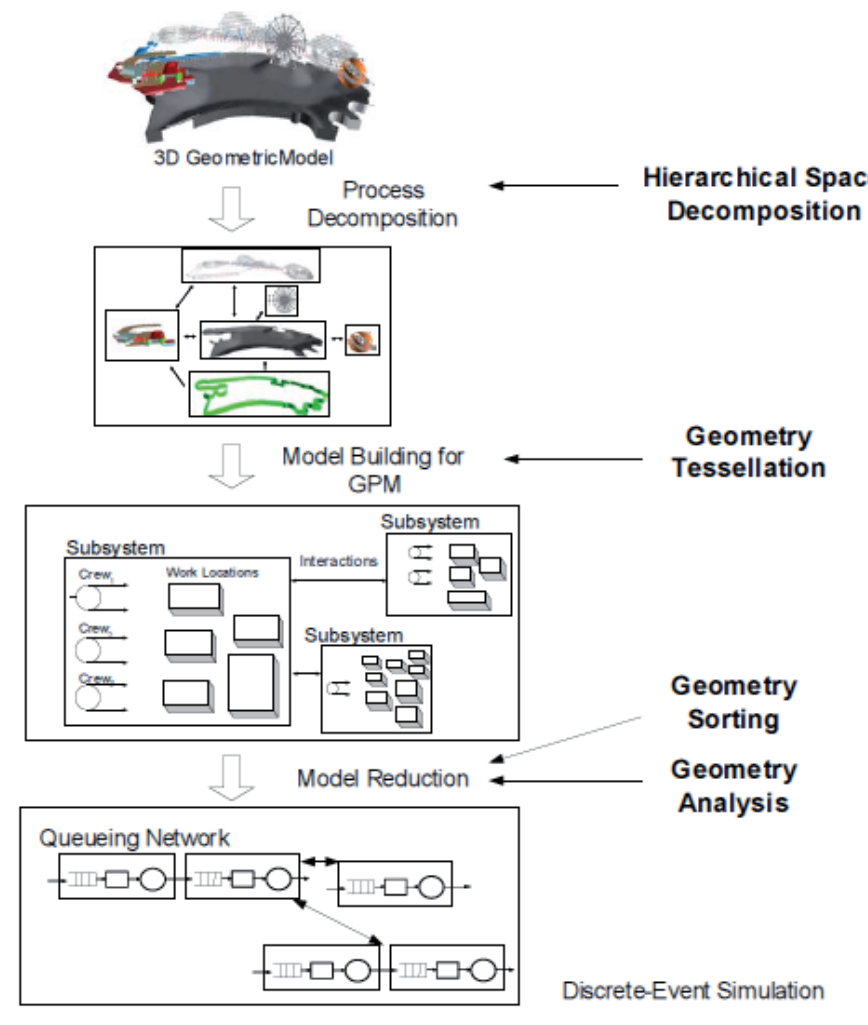

Fig. 1 Geometry based discrete-event simulation

4D modeling, which is in fact a time scheduling system based on traditional Gantt chart and a 3D construction model. The system has two levels: the estimating (attributes and quantities from the 3D building model and the Roombook extension of 3D designing software) and the Scheduling (which will use the estimated variables and the construction schedule from the Gantt chart). The scheduling will collect all available data in a database, and will generate object relation data. From this and from the Gantt chart we will have two outputs: a 4D viewer for the construction project and a material and resource diagram that will show us, when and what, and what quantity we will need on the site from the aggregation of building materials.

The restrictions of this method can easily be seen from Fig. 2: we have a 3D model, from which we will have an ideal quantity for the job. But we have to add additional quantities of the unexpected works manually, and of course, we have to assign resources and performance values manually for every single session or technology. The software will calculate the duration of a single task, and we will have a schedule based on the task durations. But the schedule will depend on our manually recorded data, and will be hard to make a change on the main technology parameters during the application.

In some cases, however, there are multiple possible schedule variants applicable. In order to find the best solution, a supplementary optimization is necessary, which should be executed before the start of the supervised construction process. Faghihi (Faghihi et al., 2014) presented a genetic algorithm-based

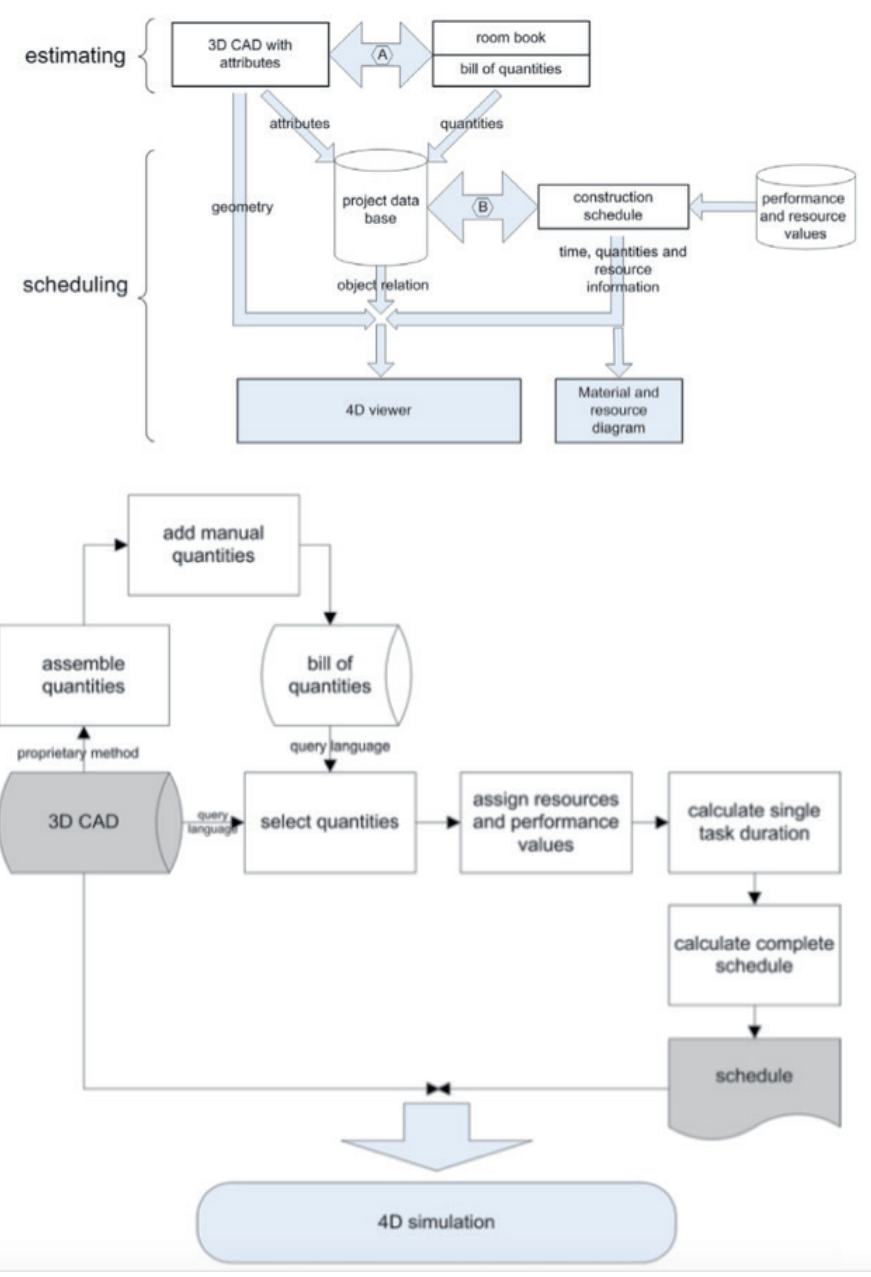

Fig. 2 3D construction model and Gantt based model

methodology for steel-structure buildings where the applied criteria are calculated from the structural stability.

In the paper of Voigtmann and her colleague (Voigtmann and Bargstädt 2010) we can also find a modeling and scheduling method for special areas of the construction process with a finer spatial resolution. A good example is the construction logistics model of cranes for material handling on construction sites. We can find some interacting modules for this application as well as shown in Fig. 3.

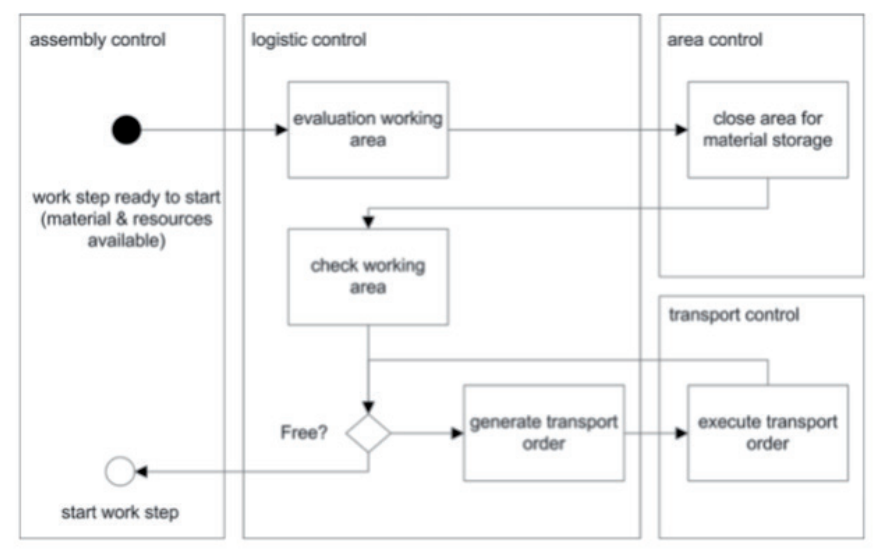

Fig. 3 Construction site material handling model 
As we can see, the logistics process model can work together with assembly control (technological process), with transportation (off-site logistics) and area control (layout management). This organization structure is closer to our research approach than any other before but has limited possibilities: Suitability information of work sequences cannot be given directly, because the work sequence (the found case) is part of the input data (defined by the technical constraints). The model can be used to generate delivery schedules, overview tables of storage areas and operation schedules for personnel and site equipment. The possible applications are training sessions and analyzing "what-if” scenarios.

We can also find some researches on transportation process modeling for constructional logistics. In their paper, Jaoua and colleagues (Joua et al., 2012), demonstrated a microscopic model of internal transport processes that is already connected with a simulation, where the latter implements emulation of the transport tasks.

A good example of examination and modeling a special area of transportation is in the paper of Sanz, and his colleagues (Sanz et al., 2013). Path planning methods relate basically a single movement from A to B of a larger scale set of movements. The latter is discussed as a Vehicle Routing Problem (VRP), where the objective function is a cost-based one.

In 2005, Navon and Shpatnitsky (Navon and Shpatnitsky 2005) demonstrated modelling methods for special construction processes, including road construction. For example, Fig. 4. shows a model of a road construction monitoring system. The input data for the model are from different sources. We will get the planned productivity and technology times from the project model (in the project model we can record the technological parameters manually). The project plan the delivery notes of the logistics process will determine the quantity of materials data. We have a resource input as well, we can have the machinery data (identification, location, post processing) from a machinery control system, the so-called Location Measurement Unit (LMU). All these data will work as an input for the Work envelope (WE) that is an elemental construction process (Work order of the Day e.g.). To activate the process monitoring, we have to have a human interaction. After the process has been started (see the ID module, we have all input data and the type of needed activity) the Conversation and Processing (CAP) module will check which needed activity is in progress on which location, and will collect data for the historical productivity database. What is unique in this model is the location and logical association in the CAP module. The disadvantage of the model (from our point of view) is that it has only manually recorded logistic data and it has only technological rules for decision making that are manually recorded as well.

Our conclusions from the models surveyed are as follows:

- We have to develop a modeling system that uses the parts of the modeling methodology of other industries.

- It has to be more adaptive and more flexible than the currently used applications of the construction industry.

- We want this modeling method to help the time-planning and technological planning of the construction project.

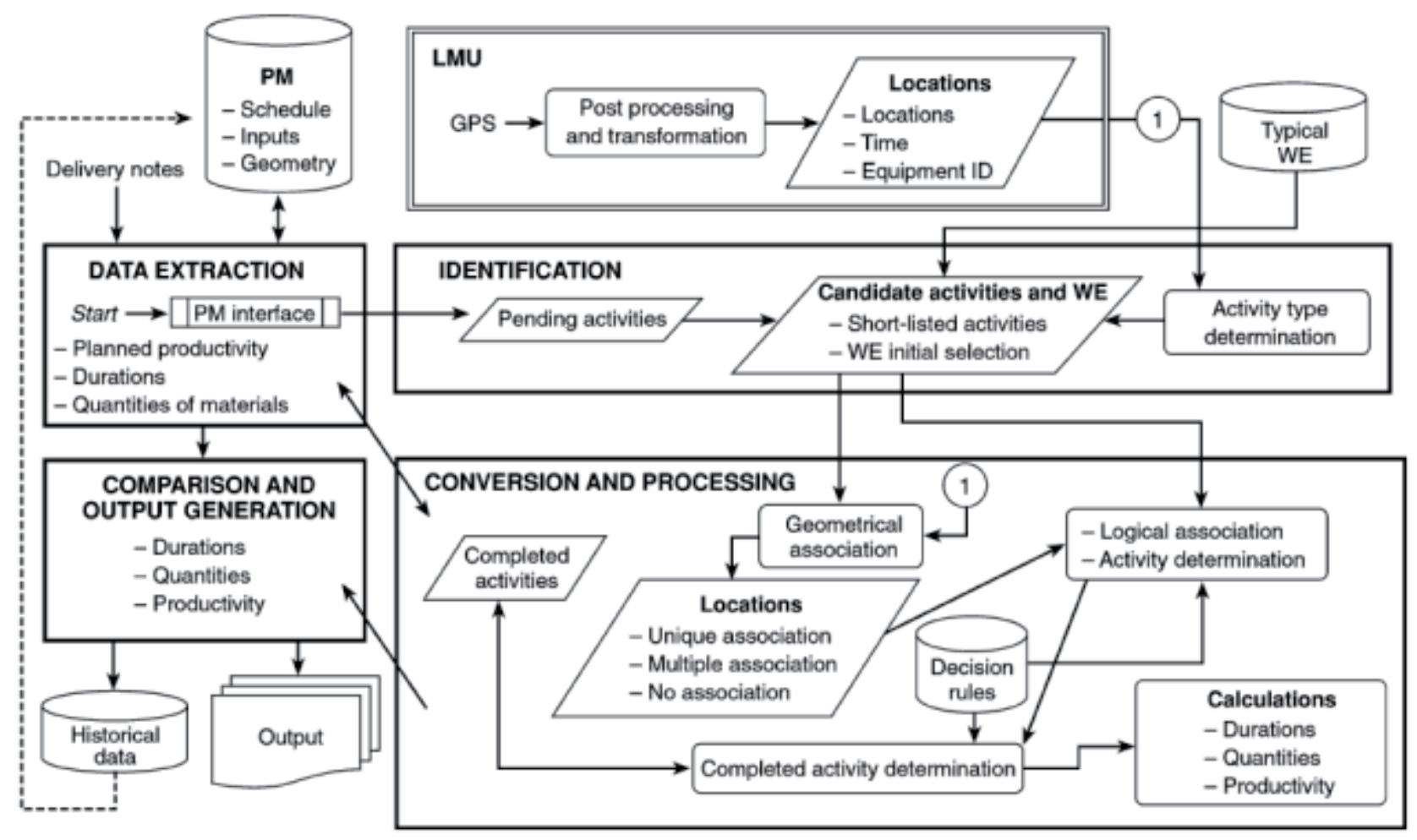

Fig. 4 Monitoring model for road construction 
Also, we need a system that can be used during the process as well, to forecast the effects of the events that had been done.

\section{Example construction logistic process}

Basically, we want to approach the construction as a complex logistics process, with some momentous and not negligible technology-based component. But the key of our developments is logistics.

The industry has some process planning software that can be used for layout planning in building construction and helps decision making in the fields of technology and time planning. But we have no tool to model the out of site processes in this case. We have to provide a solution for that.

For linear earthworks, road or railway construction we have the same situation in terms of the off-site logistics processes, but we have a continuously changing field of action: the work is conducted on various locations, and we have one or more technologies that have to be installed on a fixed position (e.g. concrete plants, crushed stone landfills). It will affect our logistic processes largely. So, as we have the situation mentioned above, we want to find a solution for the earthworks, because we will solve the off-site problems of building construction and the specialty of linear work as well.

To demonstrate the above concept, a typical (simplified) linear construction process has been chosen as an example: we have one or more excavation areas (soil mining, or creating notch), wherefrom we have to transport the soil to one or more construction areas (e.g. creating embankments or landscaping). Besides these basic processes, we can have some special processes and equipment as well, e.g. to build the pavement of the road or to build the specialties of the railway. For those technologies, we have to have logistic processes as well (e.g. mixer trucks, asphalt or crushed stone transportation vehicles). The main problem that we have to solve is that we can choose from various technologies and machines to do the same job. The most important factors are money and time: what is the reasonable cost, and what interval we have for a step.

\section{Choosing a modelling method}

As mentioned above, we have a lot of variants to make the construction process successful. But there are a lot of variables, that can modify the process, and there are a lot of them, that we can't influence: let's take the weather as a trivial example. That is the reason why we have to choose an adaptive simulation, where we can easily modify the parameters: not only the logistic ones but the technological and environmental ones as well.

For the technology selection, we can add a fuzzy based logical decision support algorithm, where we can set priorities for our variants of environment and specialties of technology. After the fuzzy-based decision support, we will have an optimal set of equipment for the job with the exact parameters we have. But during the running process, we can have difficulties, that we couldn't predict, and regarding these upcoming changes it frequently occurs - we will have to change the number of machines, or the complete technology (Balpataki et al., 2013). We have to be able to manage these changes in the model and in the simulation of the process.

As we have already introduced with our colleague Angéla Rinkács (Rinkács et al., 2014), an agent-based adaptive simulation could be the solution if we want to be flexible, which is well known in other industries. During the agent-based modeling of material flow systems, we believe that exact operation times are necessary.

The further question of modelling is, if there is a relevant parallel process which has the effect on the materials flow therefore it needs to be modelled. In container terminals, the loading units lay passively outside the handling process (stored in the ship, yard, railway carriage etc.). In case of modeling AGV systems for manufacturing there is a parallel process manufacturing - is an active one as the construction process. That means it has its own dynamics, fluctuation, which has an influence on the material flow as well, so we can use a modeling method for the construction, similar to the material flow in manufacturing.

As the paper of our colleague Péter Németh (Németh, 2013) shows roughly, when planning the traffic situation at a construction project, the following should be considered: continuous transport and supply of materials is needed at the construction site. Warehouse and inventory management provide a big savings potential, with avoiding material losses e.g. by proper storage or anti-theft protection. A good inventory management furthermore delivers transparency of stock and thus ensures higher planning reliability. Besides operational tasks, the decision makers face logistics related strategic questions also. The right decision about logistics resources leads to customer satisfaction. Our aim is to develop a tool that is able to support decision makers in logistics related questions.

On the other hand, we have an opportunity to examine logistics applications from another point of view: In case of construction logistics the first task may be to determine the inputs and the output of the proposed model.

The success of the construction project schedule can be chosen as a key factor. Therefore, schedule adherence as an output for our model is chosen. Logistics issues at a construction site affect the schedule success and quality of the project. Therefore, as an input parameter we can use logistics-related data. For example the first input can be the available space at the construction site for storing and handling goods, unloading, etc. (layout) A congested building site is likely to cause disruption in operations.

The other important parameter should be the proportion of logistics-related costs of the total cost. If not enough logisticsrelated resources are provided the schedule success is at risk.

Our third proposed parameter can be the available transport capacity at the site or at the off-site operations. Inadequate 
transport capacity will cause bottlenecks at the material and supply delivery.

With our colleague Angéla Rinkács, we have introduced interactive simulation tools (adaptive simulation) as well in the paper of WiLD 2004 (Bohács et al., 2014). The model is to simulate a process of excavation and embankment building with compaction, including the soil transportation processes. We can use an agent-based model to simulate the process. We have the following agents in our model: Loader, Truck, Truck refuel, Truck pool, Compactor, Depo, Interface, Supervisor. We can generate more agents if needed, it depends on the complexity of the process. We can use several input parameters as well, like:

- Productivity (generally influenced by the weather, or individually by machine/operator)

- Machine status (e.g. moving to destination, working, refueling)

The destination can be modified manually, but the process will determine an optimum destination for the machines.

\section{The new model}

As we examine the previously demonstrated models and methods, model structure presented in Fig. 5 is proposed.

Our model consists of four large groups: Process Control, Environmental Effects, Off-site logistics and Site process. All the first three will affect the processes on site.
Our site process model has a new point of view, as it has a double layer model. First of all, we have a geometric gridbased logistics process model for the flow of materials and other resources. Besides that we have a $3 \mathrm{D}$ construction model and Gantt-based technological 4D model. Both sub-models are connected to the process control and each other as well, so we have information flow between the sub-processes and all have info-communication with the process control. The logistics processes will distribute not only information but the necessary resources and commodity for the technological process as well.

Our process control, which will give information and commands to the site sub-processes, has a big benefit integrated, as well: We have a self-learning database and a "cyber site" module that is an adaptive simulation of the construction process with three different working orders: data collection, prediction and visualization.

In data collection mode, the module can get data from the real site processes, and it can store the data in the database module. The exact methodology will be shown in the presentation of our pilot system, but we will have technology data (excavated quantity, delivered number of pieces or delivered quantity of bulk goods, machine status and location data etc.) This module can get data also from a process control sub-module: possibility test. We can test some "what-if" scenarios in this module without trying out in the real process. This submodule will use the empirical data from the database.

GLOBAL CONSTRUCTION LOGISTICS MODEL

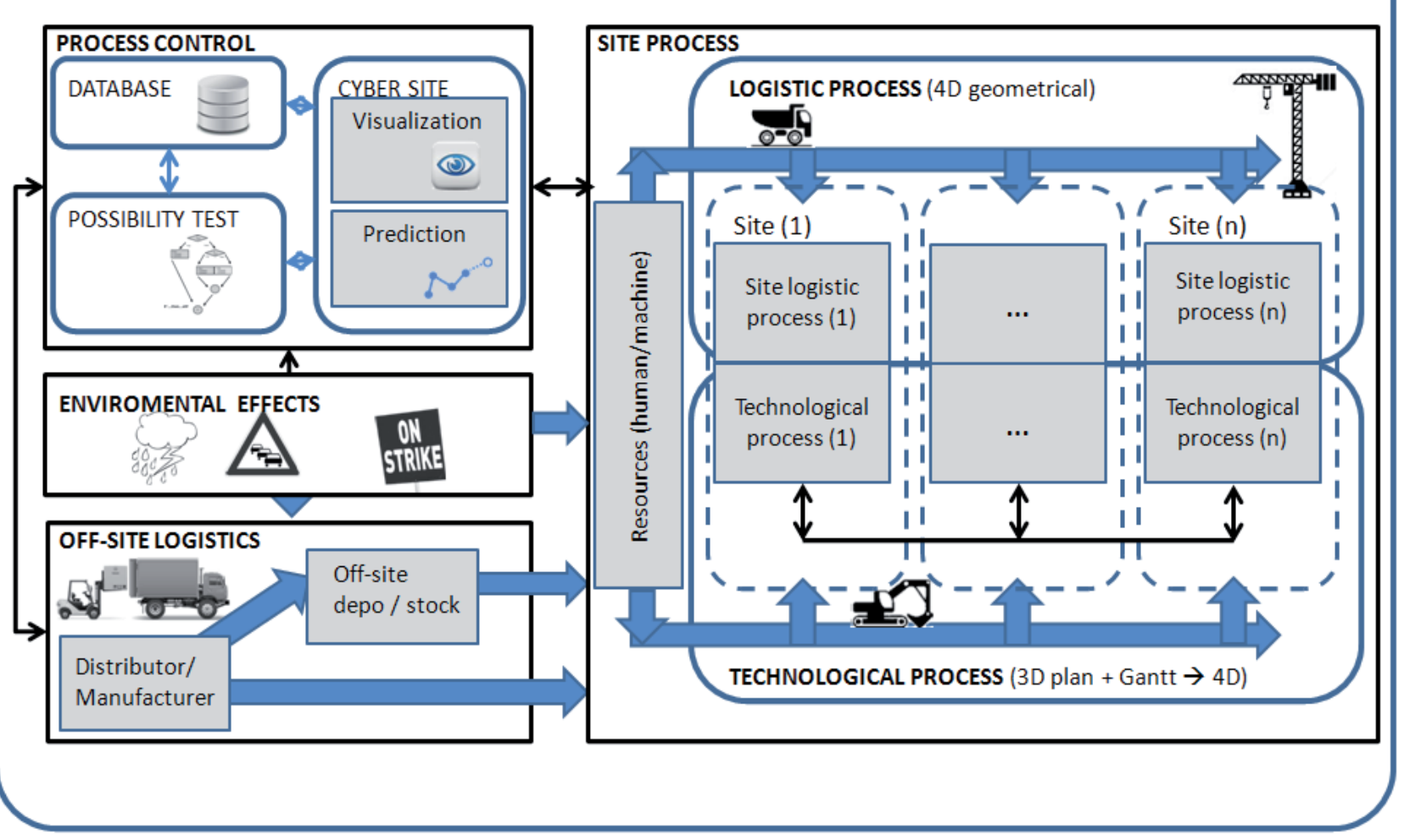

Fig. 5 The new construction logistics process model 
In visualization mode: we can follow the steps of the simulation, we can check the effects of commands.

In prediction mode, we can make long-term estimates about the effects of the changes in the parameters of the process (environmental effects, changes in logistics or technological processes, changes in resources or others).

As it can be seen in the figure, all the outer effects are connected to the process control, so we can collect data about the statistical effects of weather and other environmental effects on our technological process (e.g. cycle time or technological time changes, unexpected technological stops). As we will have collected enough large data about this, we can predict the environmental effects with appropriate (acceptable) accuracy.

\section{Pending development and future plan \\ 6.1 Developing data structure and communication}

As we have our modeling and simulation method for the construction project, we could sit back satisfied. But for the real benefit of adaptive simulation, we have to give real-time input data and real-time correction for the model: so for the proper operation we have to generate inputs from the physical process, to let the model show us, what the effects of changing those parameters will be.

A well-structured system and a well-thought-out data transfer are needed to test this connection. Of course, we will need HMIs (Human-Machine Interfaces) to control the process, and to get information on time.

First let us introduce a bit more complex composition of the "cyber site" data collection and simulation system (Fig. 6). The simulation is programmed in Simul8 software. In our example, we have split the construction process geometrically into 6 working areas (stock 1-6). We can generate several events on all geometrical areas, and we can also generate events on the machinery that all have location data too. If we make a transaction between the stock and the machine, we have made the load or unload. And of course, we have special technology events as well, as specialties of the construction technology.

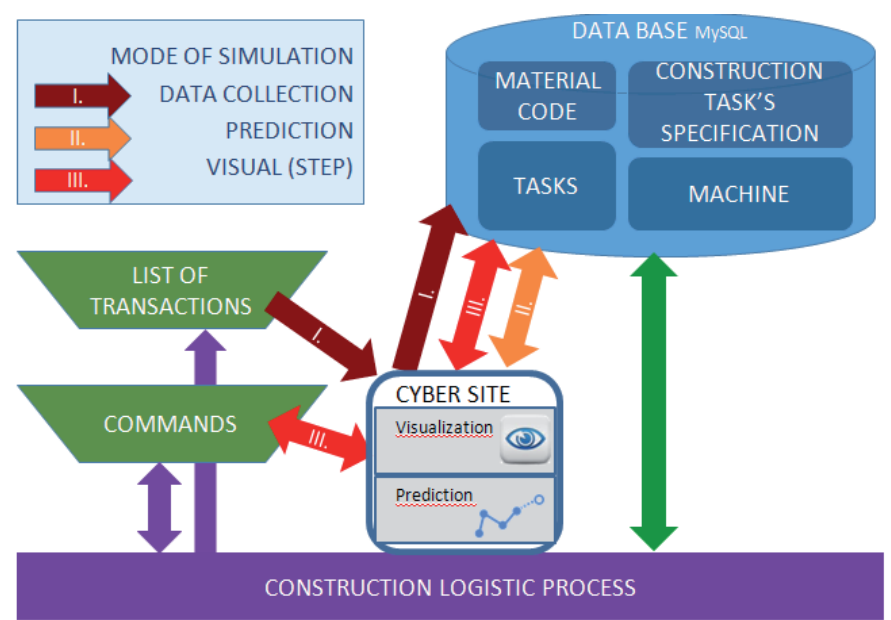

\subsection{Connecting the Model and a Pilot system}

For our test process, we have to define the input and output methods of the system as well. The operation can be explained with the operating sketch in Fig. 7. We have to have a common interface to transform all the different data from different sources (machines, data collectors, bar code readers, HMI-s and others) into a unified standard structure that we can use to fill our database up and to use as input for our simulation that generates commands from these.

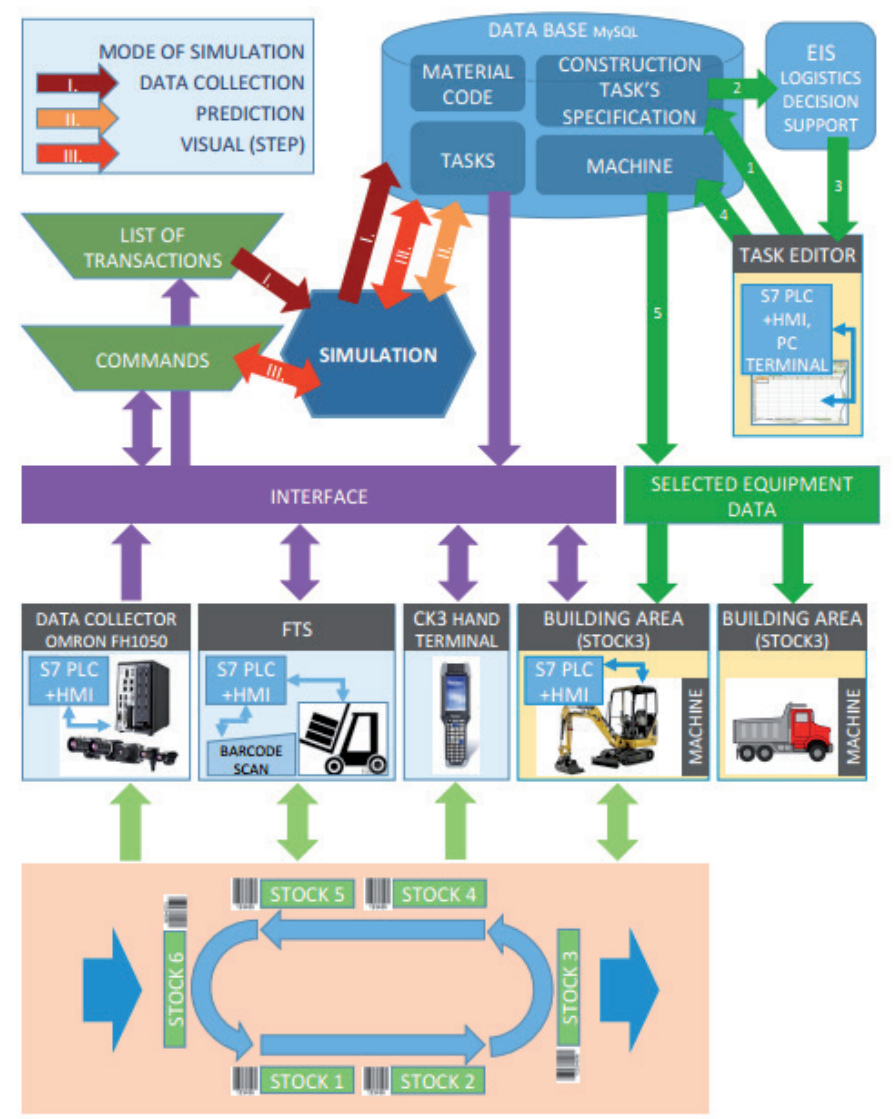

Fig. 7 Connection between the pilot system and our model

\section{Summary}

As mentioned at the beginning of this paper, we have our new model for construction processes that is double layered with technology and logistic process components, but mainly with logistic principles and methodology. From the results of the related papers and the proposed model structures, we concluded that our approach successfully integrates all the relevant modeling aspects of construction logistic process. Our next step is to test this theoretically working model in a physical test system, where we can improve and modify the model to make it more effective and more similar to the processes of the real world.

Fig. 6 "Cyber site" simulation working modes 


\section{References}

Akbaş, R. (2004) Geometry-Based Modeling and Simulation of Construction Processes. PhD Dissertation. Stanford University

Balpataki, A., Bohács, G., Odonics, B., Gyimesi, A., Rózsa, Z. (2013) Introduction of a Fuzzy-based Expert System for Selection of Materials Handling Machinery at Construction Sites. In: ISCAME conference proceeding 2013 - Debrecen, Hungary.

Bohács, G., Rinkács, A., Gyimesi, A. (2014) Entwicklung eines interaktiven Simulationstools für die Unterstützung adaptiver Modellierung. (Development of an interactive simulation tools to support adaptive modeling.) In: WILD conference article and proceeding - Leoben. (in German)

Faghihi, R. K. (2014) Construction scheduling using Genetic Algorithm based on Building Information Model. Expert Systems with Applications. 41. pp. 7565-7578 DOI: 10.1016/j.eswa.2014.05.047

Jaoua, R. G. (2012) A simulation framework for real-time fleet management in internal transport systems. Simulation Modelling Practice and Theory. 21. pp. 78-90. DOI: 10.1016/j.simpat.2011.10.003
Navon, R., Shpatnitsky, Y. (2005) A model for automated monitoring of road construction. Construction Management and Economics. 23 (9). pp. 941 951. DOI: 10.1080/01446190500183917

Németh, P. (2014) Application Possibilites of Artificial Neural Networks in the Construction Industry. In: International Conference on Computational Science and Computational Intelligence.

Rinkács, A., Gyimesi, A., Bohács, G. (2014) Adaptive Simulation of Automated Guided Vehicle Systems Using Multi Agent Based Approach for Supplying Materials. Applied Mechanics and Materials. 474.

Sanz, F. T., Gómez, E. N. E. (2013) The Vehicle Routing Problem with Limited Capacities. In: International Journal for Traffic and Transport Engineering 3. pp. 260-268. DOI:10.7708/ijtte.2013.3(3).03

Tulke, J., Hanf, J. (2007) 4D Construction Sequence Planning - New Process and Data Model. In: Conference on Information Technology in Construction - Bauhaus-University Weimar

Voigtmann, J., Bargstädt, H. J. (2010) Construction Logistics Planning by Simulation. In: Winter Simulation Conference 